Review Article

\title{
Genetic Polymorphism rs6505162 in MicroRNA-423 May Not Be Associated with Susceptibility of Breast Cancer: A Systematic Review and Meta-Analysis
}

\author{
Zhi Li, ${ }^{1}$ Jin Wang, ${ }^{2}$ Hui-bing Chen, ${ }^{3}$ Xiao-Mei Guo, ${ }^{1}$ Xiao-Ping Chen, ${ }^{4}$ Meng Wang, \\ Li-Juan Dong $\left({ }^{1},{ }^{1}\right.$ and Min-Min Zhang $\oplus^{5}$ \\ ${ }^{1}$ Department of Nursing, Zhongshan Hospital of Chinese Traditional Medicine, Zhongshan 528400, China \\ ${ }^{2}$ Department of Nephrology, Zhongshan Hospital of Chinese Traditional Medicine, Zhongshan 528400, China \\ ${ }^{3}$ Department of Anorectal, Zhongshan Hospital of Chinese Traditional Medicine, Zhongshan 528400, China \\ ${ }^{4}$ Department of Oncology, Zhongshan Hospital of Chinese Traditional Medicine, Zhongshan 528400, China \\ ${ }^{5}$ Department of Thyroid and Breast Surgery, Liuzhou People's Hospital, Liuzhou 545005, China
}

Correspondence should be addressed to Li-Juan Dong; lizhidonglijuan@163.com and Min-Min Zhang; zhmmeva@126.com

Received 24 August 2021; Revised 8 October 2021; Accepted 13 October 2021; Published 26 November 2021

Academic Editor: Yuan Seng Wu

Copyright $(2021 \mathrm{Zhi}$ Li et al. This is an open access article distributed under the Creative Commons Attribution License, which permits unrestricted use, distribution, and reproduction in any medium, provided the original work is properly cited.

\begin{abstract}
Background. MicroRNA-423 (miR-423) rs6505162 polymorphism is found to be associated with breast cancer (BC) risk. However, the results were inconsistent. This study meta-analyzed the literature on possible association between rs6505162 polymorphism and BC risk. Methods. PubMed, Embase, Google Scholar, and the Chinese National Knowledge Infrastructure (CNKI) databases were systematically searched to identify relevant studies. Meta-analyses were performed to examine the association between rs6505162 polymorphism and BC. Results. None of the five genetic models suggested a significant association between rs6505162 polymorphism and BC risk: allelic model, OR 1.02, 95\% CI 0.18-1.28, $P=0.85$; recessive model, OR 0.99, 95\% CI $0.72-1.38$, $P=0.97$; dominant model, OR 0.93, 95\% CI 0.72-1.21, $P=0.60$; homozygous model, OR 1.04, 95\% CI 0.66-1.65, $P=0.87$; and heterozygous model, OR 1.07, 95\% CI 0.90-1.28, $P=0.45$. Similar results were obtained in subgroup analyses of Asian, Chinese, and Caucasian patients. Conclusion. The available evidence suggests no significant association between rs6505162 polymorphism and $\mathrm{BC}$ risk. These conclusions should be verified in large, well-designed studies.
\end{abstract}

\section{Introduction}

Breast cancer $(\mathrm{BC})$ continues to disrupt the lives of millions of women. For many years, BC has consistently ranked among the top cancers in the women, both in terms of incidence and mortality [1]. As we all know, age, menstrual status (early menarche age and delayed menopause), reproduction (late age at first birth), genetic predisposition (higher incidence among close family members and first degree relatives in the breast cancer patients), lifestyle (saturated fat diet, alcohol excessive intake, and obesity), and so on are generally considered to be the causes of $\mathrm{BC}$ [2]. However, most causes of $\mathrm{BC}$ are not yet clearly understood. Genetic factors have been reported to play an important role in $\mathrm{BC}$ development. For instance, mutation in BRCA1 and BRCA2 and low-penetrance common genetic variants were identified as breast cancer risk factors [3]. Recent research studies have shown that one miRNA can potentially affect the expression of many genes to various degrees, and it could participate in the control of numerous metabolic pathways, including cellular growth and differentiation, suggesting that single nucleotide polymorphisms located within miRNAs can have extremely far reaching effects and may affect the development of multiple diseases, including BC [4-8]. These results indicated that miRNAs may also be risk factors for BC.

miR-423 is located in frequently amplified region of chromosome $17 \mathrm{q} 11.2$ and can produce two mature 
sequences: miR-423-3p and miR-423-5p [6]. Recent studies have shown that rs6505162: $\mathrm{C}>\mathrm{A}$, in pre-miR-423 increases risk of familial $\mathrm{BC}$ in families with a strong history of $\mathrm{BC}$ [7] and SNP rs6505162 in pre-miR-423 affects the mature miR expression, and then miR-423 may play a oncogenic role in breast tumorigenesis [8]. However, results of a recent metaanalysis including only two case-control studies on rs6505162 showed no relationship between $\mathrm{rs} 6505162$ polymorphism and $\mathrm{BC}$ risk [9]. Given the limited sample size, there is currently no consensus on whether there exists an association between rs6505162 polymorphism and BC risk.

As some new studies published, we conducted this metaanalysis of all relevant literatures to provide comprehensive and reliable insights. To the best of our knowledge, this is the first meta-analysis especially concerning rs6505162 polymorphism and BC risk, and it has the largest sample at present, compared with those published ones.

\section{Materials and Methods}

2.1. Search Strategy. All clinical and experimental casecontrol studies of polymorphisms in the miR-423 gene and BC published through May 15, 2021, were identified through systematic searches in PubMed, Embase, Google Scholar, and the Chinese National Knowledge Infrastructure (CNKI) databases, without language restrictions. The search terms used were: microRNA-423; miR-423; rs6505162; these three terms in combination with polymorphism, polymorphisms, SNP, variant, variants, variation, genotype, genetic, or mutation; and all of the above terms in combination with breast cancer, mammary cancer, or mammary adenocarcinoma. Reference lists in identified articles and reviews were also searched manually to identify additional eligible studies.

2.2. Inclusion Criteria. To be included in our review and meta-analysis, studies had to (1) have a case-control design for assessing the association between rs6505162 polymorphism and BC risk; (2) be accessible as a full-text article and report sufficient data for estimating odds ratios (ORs) with 95\% confidence intervals (CIs); (3) report genotype frequencies; and (4) involve humans rather than animal models.

2.3. Data Extraction. Two authors (ZL and LJD) independently extracted the following data from included studies: first author's family name, year of publication, ethnicity, testing methods, NOS score, $P$ value for Hardy-Weinberg equilibrium (HWE) in controls, control source, sample size, matched parameters, and numbers and genotypes of cases and controls. Discrepancies were resolved by consensus. Only those studies that met the predetermined inclusion criteria were included.

2.4. Assessment of Methodological Quality. To assess the quality of the studies included in this analysis, the Newcastle-Ottawa Scale was applied independently by two assessors (ZL and LJD) [10] (Table 1). On the 10-point Newcastle-Ottawa Scale, scores of 5-9 points (stars) are considered to indicate generally high methodological quality, while scores of $0-4$ stars are considered to indicate poor quality [11]. Any disagreements about Newcastle-Ottawa scores were resolved by other authors following a comprehensive reassessment. Only high-quality studies were included in the meta-analysis.

2.5. Statistical Analysis. Unadjusted odds ratios (ORs) with 95\% confidence intervals (CIs) were used to assess the strength of the association between rs6505162 polymorphism and BC risk based on genotype frequencies in cases and controls. The significance of pooled ORs was determined using the $Z$ test, with $P<0.05$ defined as the significance threshold. Meta-analysis was conducted using a fixed-effect model when $P>0.10$ for the $Q$ test, indicating lack of heterogeneity among studies; otherwise, a random-effect model was used. All these statistical tests were performed using Review Manager 5.3 (Cochrane Collaboration).

Publication bias was assessed using Begg's funnel plots and Egger's weighted regression in Stata 12.0 (Stata Corp., College Station, TX, USA), with $P<0.05$ considered statistically significant.

\section{Results}

3.1. Description of Studies. Figure 1 shows a flowchart illustrating the process of searching for and selecting studies. A total of 294 potentially relevant publications were identified. Of these, we excluded 277 studies during initial screening based on review of the titles and abstracts. During analysis of the full text of the remaining articles, two studies were excluded for investigating other miRNAs $[12,13]$, two studies were excluded because they were review articles $[14,15]$, and one study was excluded because it did not report precise genotypes [16].

In the end, 12 studies [7, 17-27] were included in this meta-analysis based on our search strategy and inclusion criteria. Their characteristics and genotype distributions are summarized in Tables 1 and 2, respectively. The distribution of genotypes in controls was consistent with Hardy-Weinberg equilibrium (HWE, $P>0.05)$ in all but one study [25]. The overall quality of the included studies was adequate, and the mean Newcastle-Ottawa score for the included studies was 6.75 (Table 3).

3.2. Quantitative Data Synthesis. The meta-analysis of a possible association between rs6505162 polymorphism and $\mathrm{BC}$ risk is summarized in Table 4 . Based on the total study population including 2,689 cases and 2,980 controls from 12 studies [7, 17-27], none of the five genetic models indicated a significant association: allelic model, OR 1.02, 95\% CI $0.18-1.28, P=0.85$ (Figure 2(a)); recessive model, OR 0.99, 95\% CI 0.72-1.38, $P=0.97$ (Figure 2(b)); dominant model, OR $0.93,95 \%$ CI $0.72-1.21, P=0.60$ (Figure 2(c)); homozygous model, OR 1.04, 95\% CI 0.66-1.65, $P=0.87$ 
TABLE 1: Characteristics of studies included in the meta-analysis.

\begin{tabular}{|c|c|c|c|c|c|c|c|c|c|c|c|}
\hline \multirow{2}{*}{ First author } & \multirow{2}{*}{ Year } & \multirow{2}{*}{ Ethnicity } & \multirow{2}{*}{ Country } & \multirow{2}{*}{$\begin{array}{c}\text { Cancer } \\
\text { type }\end{array}$} & \multirow{2}{*}{$\begin{array}{l}\text { Testing } \\
\text { method }\end{array}$} & \multirow{2}{*}{$\begin{array}{l}\text { NOS } \\
\text { score }\end{array}$} & \multirow{2}{*}{$\begin{array}{l}P \text { for } \\
\text { HWE }\end{array}$} & \multirow{2}{*}{$\begin{array}{l}\text { Control } \\
\text { source }\end{array}$} & \multicolumn{2}{|c|}{ Sample size $(n)$} & \multirow{2}{*}{$\begin{array}{c}\text { Matched } \\
\text { parameters }\end{array}$} \\
\hline & & & & & & & & & Cases & Controls & \\
\hline $\begin{array}{l}\text { Kontorovich } \\
\text { et al. [17] }\end{array}$ & 2010 & Caucasian & Israel & $\begin{array}{l}\text { BRCA1, } \\
\text { BRCA2 }\end{array}$ & iPLEX & 6 & 0.899 & $\mathrm{~PB}$ & 190 & 206 & Undetermined \\
\hline $\begin{array}{l}\text { Smith et al. } \\
{[18]}\end{array}$ & 2012 & Caucasian & Australia & - & HRM & 7 & 0.307 & $\mathrm{HB}$ & 179 & 174 & Age, sex, ethnicity \\
\hline Ma et al. [19] & 2013 & Asian & China & TNBC & MassArray & 7 & 0.847 & $\mathrm{HB}$ & 192 & 189 & $\begin{array}{l}\text { Age, sex, ethnicity, } \\
\text { smoking status }\end{array}$ \\
\hline He et al. [20] & 2015 & Asian & China & - & MassArray & 8 & 0.103 & $\mathrm{~PB}$ & 450 & 450 & $\begin{array}{c}\text { Age, menopausal } \\
\text { status }\end{array}$ \\
\hline $\begin{array}{l}\text { Zhang et al. } \\
\text { [21] }\end{array}$ & 2015 & Asian & China & - & MassArray & 8 & 0.847 & $\mathrm{~PB}$ & 382 & 189 & $\begin{array}{c}\text { Age, smoking } \\
\text { status }\end{array}$ \\
\hline Zhao et al. [22] & 2015 & Asian & China & - & Sequencing & 6 & 0.847 & $\mathrm{~PB}$ & 114 & 189 & Undetermined \\
\hline $\begin{array}{l}\text { Morales et al. } \\
\text { [7] }\end{array}$ & 2016 & Caucasian & Chile & - & TaqMan & 6 & 0.700 & $\mathrm{HB}$ & 440 & 807 & $\begin{array}{c}\text { Age, } \\
\text { socioeconomic } \\
\text { strata }\end{array}$ \\
\hline Saedi et al. [23] & 2017 & Asian & Iran & - & PCR-RFLP & 6 & 0.196 & $\mathrm{HB}$ & 353 & 353 & Undetermined \\
\hline $\begin{array}{l}\text { Tran Thi et al. } \\
\text { [24] }\end{array}$ & 2018 & Asian & Vietnam & - & HRM & 6 & 0.071 & $\mathrm{~PB}$ & 106 & 116 & Undetermined \\
\hline Mir et al. [25] & 2018 & Asian & $\begin{array}{l}\text { Saudi } \\
\text { Arabia }\end{array}$ & - & ARMS-PCR & 7 & $<0.001$ & $\mathrm{~PB}$ & 124 & 100 & Sex \\
\hline Mir et al. [26] & 2019 & Asian & $\begin{array}{l}\text { Saudi } \\
\text { Arabia }\end{array}$ & - & ARMS-PCR & 7 & 0.152 & $\mathrm{~PB}$ & 30 & 30 & Sex \\
\hline $\begin{array}{l}\text { Pourmoshir } \\
\text { et al. [27] }\end{array}$ & 2020 & Asian & Iran & - & ARMS-PCR & 7 & 0.206 & $\mathrm{~PB}$ & 153 & 153 & Sex \\
\hline
\end{tabular}

Abbreviations: BRCA1, breast cancer type 1 susceptibility gene; BRCA2, breast cancer type 2 susceptibility gene; TNBC, triple-negative breast cancer; HB, hospital-based source of control; PB, population-based source of control; PCR, polymerase chain reaction; RFLP, restriction fragment length polymorphism; HRM, high-resolution melting; ARMS, amplification refractory mutation system; HWE, Hardy-Weinberg equilibrium.

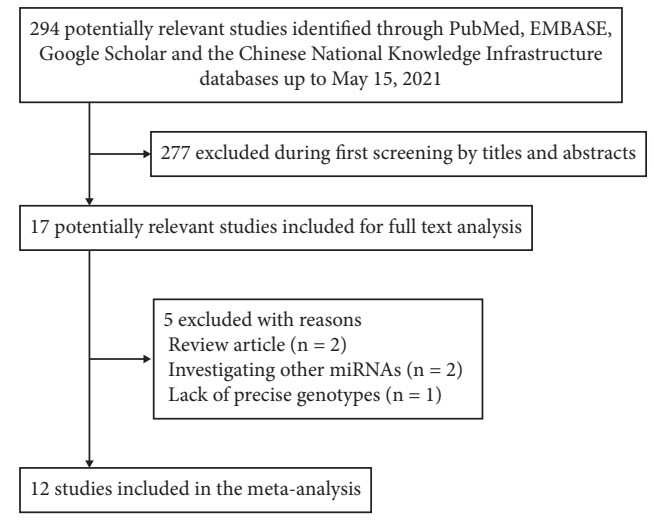

Figure 1: Flowchart showing search strategies, selection criteria, and included studies.

(Figure 2(d)); and heterozygous model, OR 1.07, 95\% CI $0.90-1.28, P=0.45$ (Figure 2(e)).

Next we meta-analyzed data for subgroups based on ethnicity. Meta-analysis of 9 studies [19-27] involving 1,880 Asian cases and 1,793 Asian controls showed no evidence of a significant association rs6505162 polymorphism and $\mathrm{BC}$ risk in any of the five genetic models (Table 4): allelic model, $\mathrm{OR}=1.09,95 \%$ CI $0.82-1.44, P=0.56$; recessive model, $\mathrm{OR}=1.10,95 \% \mathrm{CI}=0.75-1.61, P=0.64$; dominant model, $\mathrm{OR}=0.81, \quad 95 \% \quad \mathrm{CI}=0.63-1.03, \quad P=0.09 ;$ homozygous model, $\mathrm{OR}=1.20,95 \% \mathrm{CI}=0.69-2.08, P=0.52$; and heterozygous model, $\mathrm{OR}=1.20,95 \% \mathrm{CI}=0.92-1.56, P=0.18$.

Similarly, no evidence of an association was identified in meta-analysis of 4 studies [19-22] involving 1,138
Chinese cases and 1,017 Chinese controls (Table 4): allelic model, $\mathrm{OR}=1.12,95 \% \mathrm{CI}=0.97-1.30, P=0.13$; recessive model, $\mathrm{OR}=1.13,95 \% \mathrm{CI}=0.95-1.35, P=0.18$; dominant model, $\mathrm{OR}=0.81,95 \% \mathrm{CI}=0.54-1.22, P=0.32$; homozygous model, $\mathrm{OR}=1.29,95 \% \mathrm{CI}=0.85-1.95, P=0.24$; and heterozygous model, $\mathrm{OR}=1.15,95 \% \mathrm{CI}=0.75-1.76$, $P=0.53$.

Also, no evidence of an association was identified in meta-analysis of 3 studies [7, 17, 18] involving 809 Caucasian cases and 1,187 Chinese controls (Table 4): allelic model, $\mathrm{OR}=0.87,95 \% \mathrm{CI}=0.58-1.31, P=0.51$; recessive model, $\mathrm{OR}=0.75,95 \% \mathrm{CI}=0.38-1.48, P=0.41$; dominant model, $\mathrm{OR}=1.11,95 \% \mathrm{CI}=0.74-1.66, P=0.63$; homozygous model, $\mathrm{OR}=0.75,95 \% \mathrm{CI}=0.33-1.70, P=0.49$; and 


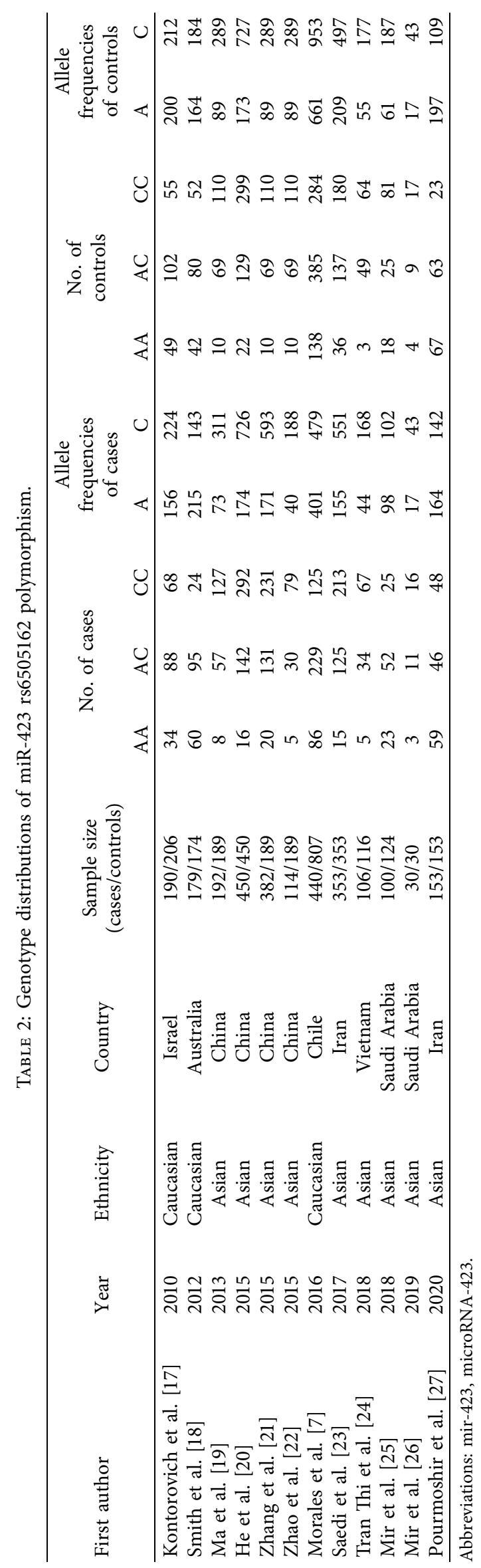




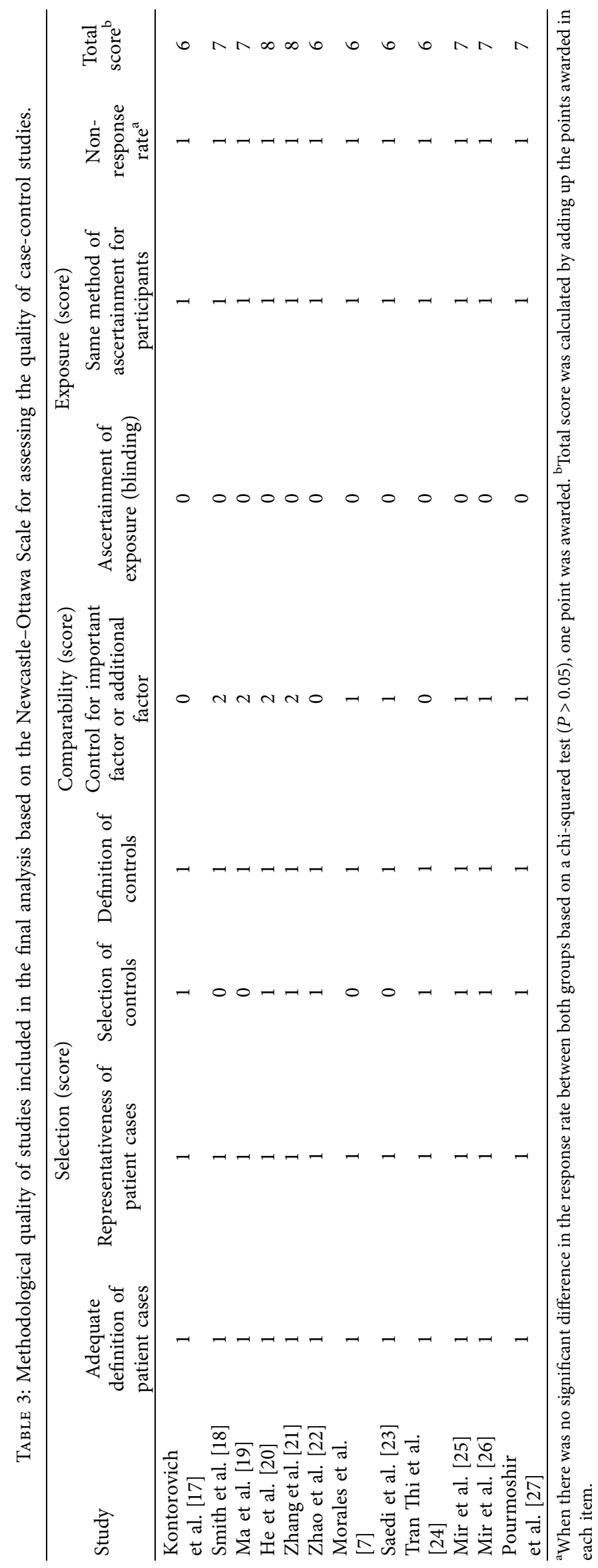


TABLE 4: Overall meta-analysis of the association between breast cancer and miR-423 rs6505162 polymorphism.

\begin{tabular}{|c|c|c|c|c|c|c|}
\hline \multirow{2}{*}{ Genetic model } & \multirow{2}{*}{ OR $[95 \% \mathrm{CI}]$} & \multirow{2}{*}{$\mathrm{Z}$ ( $P$ value $)$} & \multicolumn{3}{|c|}{ Heterogeneity of study design } & \multirow{2}{*}{ Analysis mode } \\
\hline & & & $x^{2}$ & $\mathrm{~d} f(P$ value $)$ & $I^{2}(\%)$ & \\
\hline \multicolumn{7}{|c|}{ Mir-423 rs6505162 in total population from 12 case control studies $[7,17-27](2,689$ cases and 2,980 controls) } \\
\hline Allelic model (C-allele vs. A-allele) & $1.02[0.81,1.28]$ & $0.19(0.85)$ & 73.30 & $11(<0.001)$ & 85 & Random \\
\hline Recessive model (CC vs. $\mathrm{AC}+\mathrm{AA})$ & $0.99[0.72,1.38]$ & $0.03(0.97)$ & 82.60 & $11(<0.001)$ & 87 & Idom \\
\hline Dominant model (AA vs. AC $+\mathrm{CC}$ ) & $0.93[0.72,1.21]$ & $0.52(0.60)$ & 21.40 & $11(0.03)$ & 49 & Random \\
\hline Homozygous model (CC vs. AA) & $1.04[0.66,1.65]$ & $0.17(0.87)$ & 54.28 & $11(<0.001)$ & 80 & Random \\
\hline Heterozygous model (AC vs. AA) & $1.07[0.90,1.28]$ & $0.76(0.45)$ & 11.49 & $11(0.40)$ & 4 & Fixed \\
\hline \multicolumn{7}{|c|}{ Mir-423 rs6505162 in Asian population from 9 case-control studies [19-27] (1,880 cases and 1,793 controls) } \\
\hline Allelic model (C-allele vs. A-allele) & $1.09[0.82,1.44]$ & $0.58(0.56)$ & 47.22 & $8(<0.001)$ & 83 & Random \\
\hline Recessive model (CC vs. AC + AA) & $1.10[0.75,1.61]$ & $0.47(0.64)$ & 55.74 & $8(<0.001)$ & 86 & Random \\
\hline Dominant model (AA vs. AC + CC) & $0.81[0.63,1.03]$ & $1.72(0.09)$ & 11.91 & $8(0.16)$ & 33 & Fixed \\
\hline Homozygous model (CC vs. AA) & $1.20[0.69,2.08]$ & $0.64(0.52)$ & 29.58 & $8(<0.001)$ & 73 & Random \\
\hline Heterozygous model (AC vs. AA) & $1.20[0.92,1.56]$ & $1.35(0.18)$ & 9.04 & $8(0.34)$ & 11 & Fixed \\
\hline \multicolumn{7}{|c|}{ Mir-423 rs6505162 in Chinese population from 4 case-control studies [19-22] (1,138 cases and 1,017 controls) } \\
\hline Allelic model (C-allele vs. A-allele) & $1.12[0.97,1.30]$ & $1.50(0.13)$ & 3.37 & $3(0.34)$ & 11 & Fixed \\
\hline Recessive model (CC vs. AC + AA) & $1.13[0.95,1.35]$ & $1.35(0.18)$ & 4.99 & $3(0.17)$ & 40 & Fixed \\
\hline Dominant model (AA vs. $\mathrm{AC}+\mathrm{CC}$ ) & $0.81[0.54,1.22]$ & $1.00(0.32)$ & 0.39 & 94) & 0 & Fixed \\
\hline Homozygous model (CC vs. AA) & $1.29[0.85,1.95]$ & $1.19(0.24)$ & 0.36 & 3( & 0 & Fixed \\
\hline Heterozygous model (AC vs. AA) & $1.15[0.75,1.76]$ & $0.62(0.53)$ & 1.10 & $3(0.78)$ & 0 & Fixed \\
\hline \multicolumn{7}{|c|}{ Mir-423 rs6505162 in Caucasian population from 3 case-control studies $[7,17,18]$ (809 cases and 1,187 controls) } \\
\hline Allelic model (C-allele vs. A-allele) & $0.87[0.58,1.31]$ & $0.66(0.51)$ & 16.19 & $2(<0.001)$ & 88 & \\
\hline Recessive model (CC vs. AC+ AA) & $0.75[0.38,1.48]$ & $0.82(0.41)$ & 17.51 & $2(<0.001)$ & 89 & Random \\
\hline Dominant model (AA vs. AC + CC) & $1.11[0.74,1.66]$ & $0.49(0.63)$ & 5.80 & $2(0.06)$ & 66 & Random \\
\hline Homozygous model (CC vs. AA) & $0.75[0.33,1.70]$ & $0.70(0.49)$ & 16.17 & $2(<0.001)$ & 88 & Random \\
\hline Heterozygous model (AC vs. AA) & $0.98[0.77,1.24]$ & $0.20(0.84)$ & 1.25 & $2(0.54)$ & 0 & Fixed \\
\hline \multicolumn{7}{|c|}{ Mir-423 rs6505162 in female population from 5 case-control studies $[18-21,27](1,356$ cases and 1,155 controls $)$} \\
\hline Allelic model (C-allele vs. A-allele) & $1.05[0.77,1.42]$ & $0.29(0.78)$ & 21.54 & $4(<0.001)$ & 81 & Random \\
\hline Recessive model ( $\mathrm{CC}$ vs. $\mathrm{AC}+\mathrm{AA})$ & $1.06[0.66,1.71]$ & $0.24(0.81)$ & 27.48 & $4(<0.001)$ & 85 & Random \\
\hline Dominant model (AA vs. AC $+\mathrm{CC}$ ) & $1.00[0.77,1.30]$ & $0.02(0.99)$ & 5.86 & $4(0.21)$ & 32 & Fixed \\
\hline Homozygous model (CC vs. AA) & $1.08[0.52,2.27]$ & $0.21(0.83)$ & 21.54 & $4(<0.001)$ & 81 & Random \\
\hline Heterozygous model (AC vs. AA) & $0.95[0.72,1.26]$ & $0.34(0.73)$ & 2.34 & $4(0.67)$ & 0 & Fixed \\
\hline
\end{tabular}

Abbreviations: mir-423, microRNA-423; OR, odds ratios; 95\% CI, 95\% confidence interval.

\begin{tabular}{|c|c|c|c|c|c|c|c|c|c|}
\hline \multirow{3}{*}{$\begin{array}{l}\text { Study or subgroup } \\
\text { Kontorovich et al. } 2010\end{array}$} & \multicolumn{2}{|c|}{$\begin{array}{c}\text { Cases } \\
\text { Events Total }\end{array}$} & \multicolumn{2}{|c|}{$\begin{array}{l}\text { Controls } \\
\end{array}$} & \multirow{2}{*}{$\begin{array}{c}\text { Weight } \\
(\%)\end{array}$} & \multirow{2}{*}{$\begin{array}{c}\text { Odds Ratio } \\
\text { M-H, Random, } 95 \% \mathrm{CI} \\
\end{array}$} & \multirow{2}{*}{\multicolumn{3}{|c|}{$\begin{array}{c}\text { Odds Ratio } \\
\text { M-H, Random, } 95 \% \text { CI }\end{array}$}} \\
\hline & & & & & & & & & \\
\hline & 224 & 380 & 212 & 412 & 9.0 & $1.35[1.01,1.79]$ & & 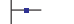 & \\
\hline Matte al. 2013 & $\begin{array}{l}143 \\
311\end{array}$ & $\begin{array}{l}358 \\
384\end{array}$ & $\begin{array}{l}184 \\
289 \\
289\end{array}$ & $\begin{array}{l}348 \\
378\end{array}$ & 8.8 & $\begin{array}{l}0.59[0.44,0.80] \\
1.31[0.93,1.86]\end{array}$ & & - & \\
\hline Heet al. 2015 & 726 & & 727 & 900 & 9.4 & $0.99[0.79,1.25]$ & & & \\
\hline & 593 & & & & 89 & $1.07[080,143$ & & & \\
\hline Zhao et al. 2015 & 188 & 228 & 289 & 378 & 7.7 & $1.45[0.95,2.19]$ & & & \\
\hline Morales et al. 2016 & 479 & 880 & 953 & 1614 & 9.9 & $0.83[0.70,0.98]$ & $\rightarrow$ & & \\
\hline Saedi et al. 2017 & 551 & 706 & 497 & 706 & 9.3 & $1.49[1.18,1.90]$ & & $\rightarrow$ & \\
\hline Tran thi et al. 2018 & 168 & 212 & 177 & 232 & 7.4 & $1.19[0.76,1.86]$ & & & \\
\hline Mir et al. 2018 & 102 & 200 & 187 & 248 & 7.9 & $0.34[0.23,0.51]$ & & & \\
\hline $\begin{array}{l}\text { Mir et al. } 2019 \\
\text { Por }\end{array}$ & & 60 & 43 & 60 & 4.6 & $1.00[0.45,2.21]$ & & & \\
\hline Pourmoshir et al. 2020 & 142 & 306 & 109 & 306 & 8.6 & $1.56[1.13,2.16]$ & & $=$ & \\
\hline Total $(95 \% \mathrm{CI})$ & & 5378 & & 5960 & 100.0 & $1.02[0.81,1.28]$ & & & \\
\hline Total events & 3670 & & 3956 & & & & & & \\
\hline \multicolumn{7}{|c|}{$\begin{array}{l}\text { Heterogeneity: tau }{ }^{2}=0.13, \mathrm{chi}^{2}=73.30, \mathrm{df}=11(P<0.00001) ; I^{2}=85 \% \\
\text { Test for overall effect: } Z=0.19(P=0.85)\end{array}$} & 0.2 & 2 & 5 \\
\hline
\end{tabular}

(a)

\begin{tabular}{|c|c|c|c|c|c|c|c|c|c|c|}
\hline \multirow{3}{*}{$\begin{array}{l}\text { Study or subgroup } \\
\text { Kontorovich et al. } 2010\end{array}$} & \multicolumn{2}{|c|}{$\begin{array}{c}\text { Cases } \\
\text { Events Total }\end{array}$} & \multicolumn{2}{|c|}{$\begin{array}{c}\text { Controls } \\
\text { Events Total } \\
\end{array}$} & \multirow{2}{*}{$\begin{array}{c}\begin{array}{c}\text { Weight } \\
(\%)\end{array} \\
11.6\end{array}$} & \multirow{2}{*}{$\begin{array}{c}\begin{array}{c}\text { Odds Ratio } \\
\text { M-H, Random, } 95 \% \mathrm{CI}\end{array} \\
0.70[0.43,1.14]\end{array}$} & \multicolumn{4}{|c|}{$\begin{array}{c}\text { Odds Ratio } \\
\text { M-H, Random, } 95 \% \text { CI }\end{array}$} \\
\hline & 34 & 190 & 49 & 206 & & & & & & \\
\hline & 60 & 179 & 42 & 174 & 12.1 & $1.58[0.99,2.52]$ & & & & \\
\hline Ma et al. 2013 & 8 & 192 & 10 & 189 & 5.4 & $0.78[0.30,2.02]$ & & & & \\
\hline He et al. 2015 & 16 & 450 & 22 & 450 & 8.7 & $0.72[0.37,1.38]$ & & $\rightarrow$ & & \\
\hline Zhang et al. 2015 & 20 & 382 & 10 & 189 & 7.1 & $0.99[0.45,2.16]$ & & & & \\
\hline Zhao et al. 2015 & 5 & 114 & 10 & 189 & 4.4 & $0.82[0.27,2.47]$ & & & & \\
\hline Morales et al. 2016 & 86 & 440 & 138 & 807 & 15.7 & $1.18[0.87,1.59]$ & & & & \\
\hline $\begin{array}{l}\text { Saedi et al. } 2017 \\
\text { Trantion }\end{array}$ & 15 & 353 & 36 & 353 & 9.3 & $0.39[0.21,0.73]$ & & $=$ & & \\
\hline $\begin{array}{l}\text { Tran thi et al. } 2018 \\
\text { Mir et al. } 2018\end{array}$ & $\begin{array}{c}5 \\
23\end{array}$ & 106 & $\begin{array}{c}3 \\
18\end{array}$ & ${ }^{116}$ & 2.7 & $\begin{array}{l}1.86[0.43,8.00] \\
1.76[0.89 .341\end{array}$ & & & & \\
\hline $\begin{array}{l}\text { Mir et al. } 2018 \\
\text { Mir et al. } 2019\end{array}$ & ${ }_{3}^{23}$ & $\begin{array}{l}100 \\
30\end{array}$ & $\begin{array}{c}18 \\
4\end{array}$ & $\begin{array}{l}124 \\
30\end{array}$ & $\begin{array}{l}8.4 \\
2.3\end{array}$ & $\begin{array}{l}1.76[0.89,3.48] \\
0.72[0.15,3.54]\end{array}$ & & & $=$ & \\
\hline Pourmoshir et al. 202 & 59 & 153 & 67 & 153 & 12.3 & $0.81[0.51,1.27]$ & & - & & \\
\hline Total $(95 \% \mathrm{CI})$ & & 2689 & & 2980 & 100.0 & $0.93[0.72,1.21]$ & & & & \\
\hline \multirow{2}{*}{\multicolumn{7}{|c|}{$\begin{array}{l}\text { Total events } \quad 334 \quad 409 \\
\text { Heterogeneity: } \text { tau }^{2}=0.09 \text {, }{ }^{2}=21.40, \mathrm{df} f=11(P=0.03) ; I^{2}=49 \% \\
\text { Test for overall effect: } Z Z=0.52(P=0.60)\end{array}$}} & & & & \\
\hline & & & & & & & 0.05 & 0.2 & 5 & 20 \\
\hline
\end{tabular}

(c)

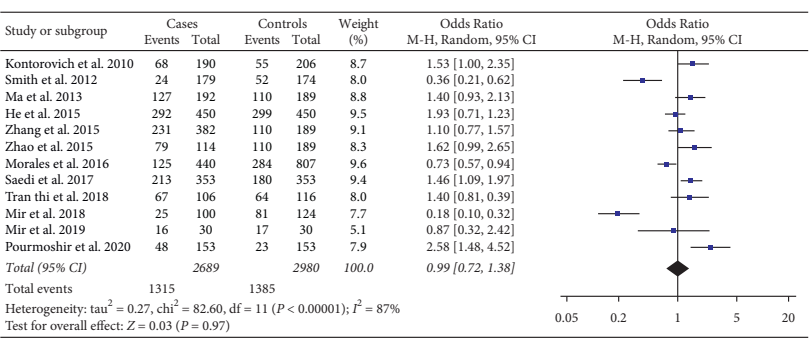

(b)

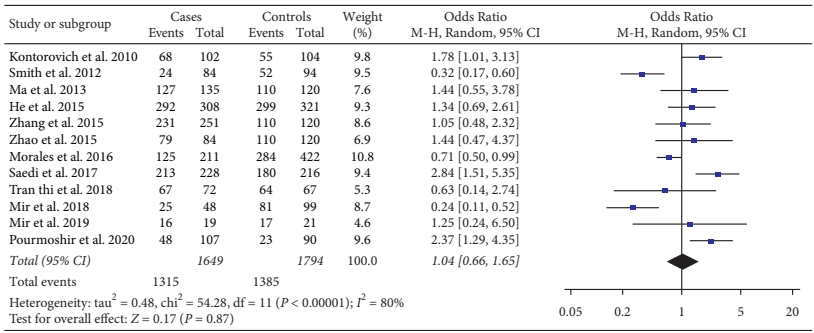

(d)

Figure 2: Continued. 


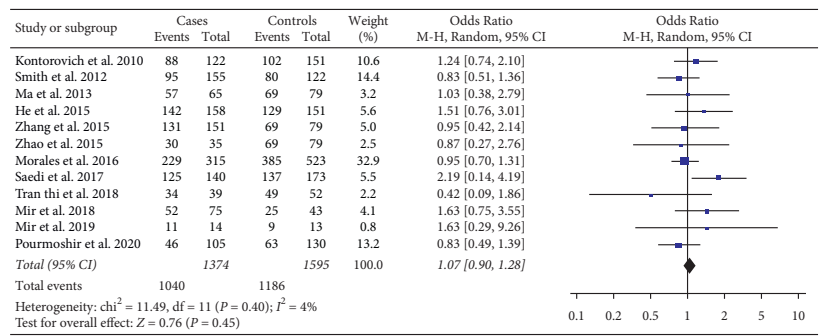

(e)

FIGURE 2: Forest plot showing the relationship between microRNA-423 rs6505162 polymorphism and breast cancer risk in total population according to different genetic models: (a) allelic model (G-allele vs. A-allele), (b) recessive model (GG vs. AG + AA), (c) dominant model (AA vs. AG + GG), (d) homozygous model (GG vs. AA), and (e) heterozygous model (AG vs. AA). Abbreviations: CI, confidence interval; df, degree of freedom; MH, Mantel-Haenszel.

heterozygous model, $\mathrm{OR}=0.98,95 \% \quad \mathrm{CI}=0.77-1.24$, $P=0.84$.

Lastly, no evidence of an association was identified in meta-analysis of 5 studies [18-21, 27] involving 1,356 female cases and 1,155 female controls (Table 4): allelic model, $\mathrm{OR}=1.05,95 \% \mathrm{CI}=0.77-1.42, P=0.78$; recessive model, $\mathrm{OR}=1.06,95 \% \mathrm{CI}=0.66-1.71, P=0.81$; dominant model, $\mathrm{OR}=1.00,95 \% \mathrm{CI}=0.77-1.30, P=0.99$; homozygous model, $\mathrm{OR}=1.08,95 \% \mathrm{CI}=0.52-2.27, P=0.83$; and heterozygous model, $\mathrm{OR}=0.95,95 \% \mathrm{CI}=0.72-1.26$, $P=0.73$.

3.3. Sensitivity Analysis. The robustness of the meta-analysis of 12 studies examining a possible association between rs6505162 polymorphism and BC risk was assessed by repeating the meta-analysis after excluding a study [25] in which the $P$ value associated with HWE was less than 0.05 . Deleting these data from the meta-analysis did not alter the results obtained using any of the five genetic models, whether for the entire study population or the Asian population.

3.4. Publication Bias. Potential publication bias in this metaanalysis was assessed using Begg's funnel plot and Egger's test. In any of the five genetic models, respectively, no obvious asymmetry was observed in Begg's funnel plots (Figures 3(a), 3(c), 3(e), 3(g), and 3(i)) and Egger's test of rs6505162 polymorphism (Figures 3(b), 3(d), 3(f), 3(h), and 3(j)). $P$ values for Begg's funnel plots and Egger's tests were all greater than 0.05 . These results suggest no potential publication bias.

\section{Discussion}

In order to investigate the relationship between rs6505162 polymorphism and $\mathrm{BC}$ risk, a few recent meta-analyses $[9,28-30]$ have reported their findings. However, their results were inconsistent. Meta-analysis by Chen et al. [28] with 16 case-control studies included suggested that rs6505162 polymorphism might be associated with a reduced risk of cancers but not with BC risk in subgroup analysis of 5 case-control studies. Meta-analysis by Zhang et al. [29] with 6 case-control studies included suggested that a significantly decreased cancer risk was observed in lung cancer for rs6505162 but not in BC risk. Meta-analysis by Li et al. [30] with 8 case-control studies included suggested rs6505162 decreases the risk of cancer, showing that it is the protective factor of cancer. But subgroup analysis for BC risk was not performed.

Those previous meta-analyses did not specially focus on $\mathrm{BC}$, much less on BC by subgroup analysis by ethnicity. In order to evaluate available evidence on the possible association between rs6505162 polymorphism in miR-423 promoter and BC risk, a more detailed meta-analysis was performed. Results showed that miR-423 rs6505162 might not be associated with BC risk, regardless of ethnicity. Even though our results supported previous studies [28, 29], given larger sample with 12 case-control studies included, ours should be more convincing.

Although null results were obtained in the current study on rs6505162 polymorphism with larger sample, we really hope they would provide a reference for future studies. Nonetheless, the work still has several limitations that may affect interpretation of the results. Firstly, the $P$ value for HWE in one study [25] was less than 0.05, making these study populations not being representative of the broader target population. Nevertheless, sensitivity analyses showed that deleting the study did not alter the results. Secondly, the studies may be subject to performance bias, attrition bias, and reporting bias, although Newcastle-Ottawa scores were more than 5 for all studies, indicating high quality. Thirdly, additional confounding factors such as age, gender, and tumor status may affect the results. In order to reduce the effect of those confounding factors above on the results, we have tried our best to make stratified analysis based on those factors. In the end, only gender could be taken into account. A total of 5 case-control studies [18-21, 27] of which the patients were all definitely female were selected to investigate the relationship between rs6505162 polymorphism and BC risk on females. Nevertheless, these studies either did not report age and tumor status or aggregated them in different ways, resulting in a failure to include them in the metaanalysis. Lastly, methods used to test for polymorphisms were not uniform and they varied in sensitivity and specificity, which may reduce the robustness of the meta-analysis. 


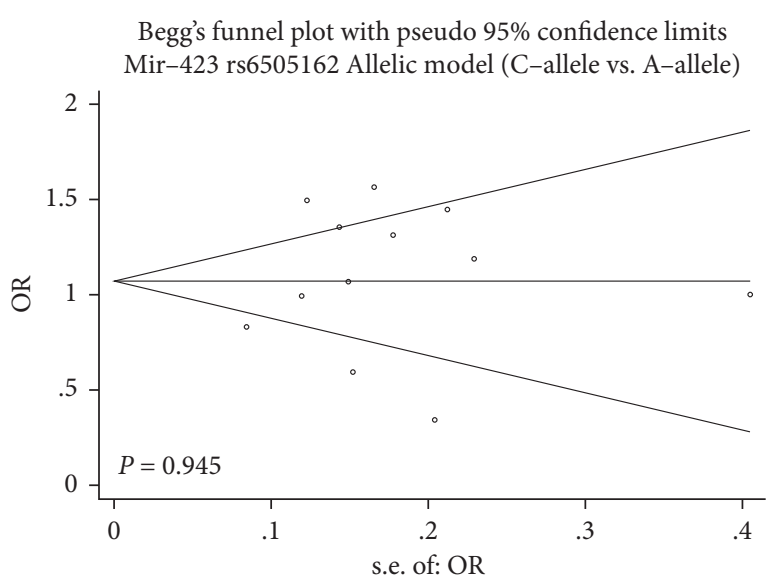

(a)

Begg's funnel plot with pseudo 95\% confidence limits Mir-423 rs6505162 Recessive model (CC vs. AC + AA)

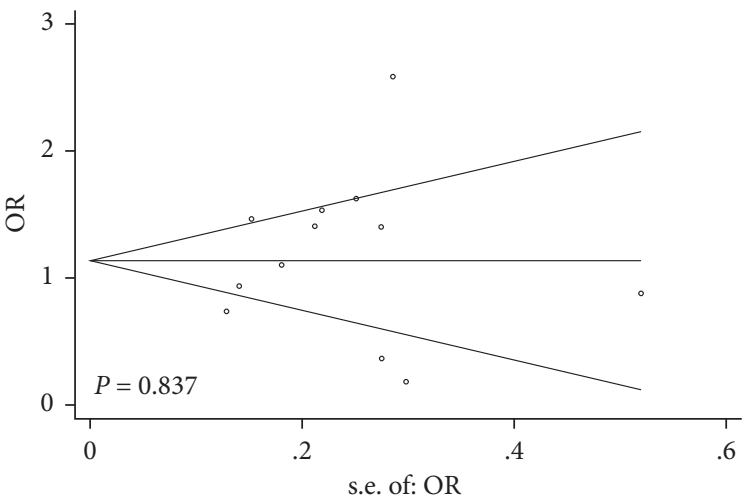

(c)

Begg's funnel plot with pseudo 95\% confidence limits Mir-423 rs6505162 Dominant model (AA vs. AC + CC)

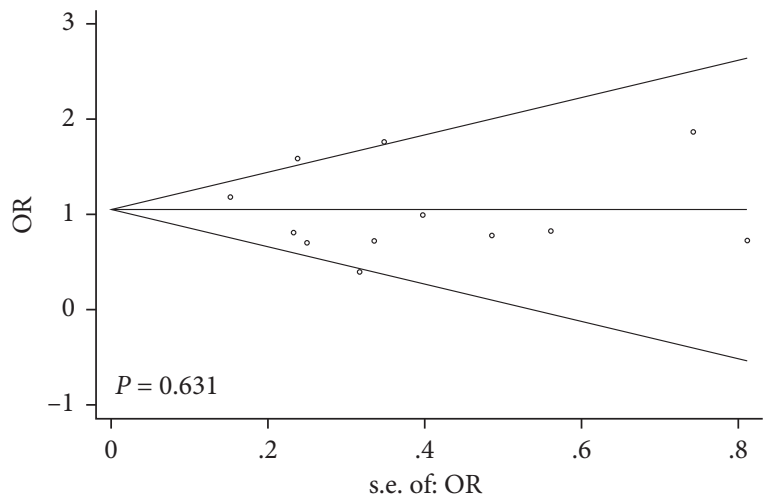

(e)
Egger's publication bias plot Mir-423 rs6505162 Allelic model (C-allele vs. A-allele)

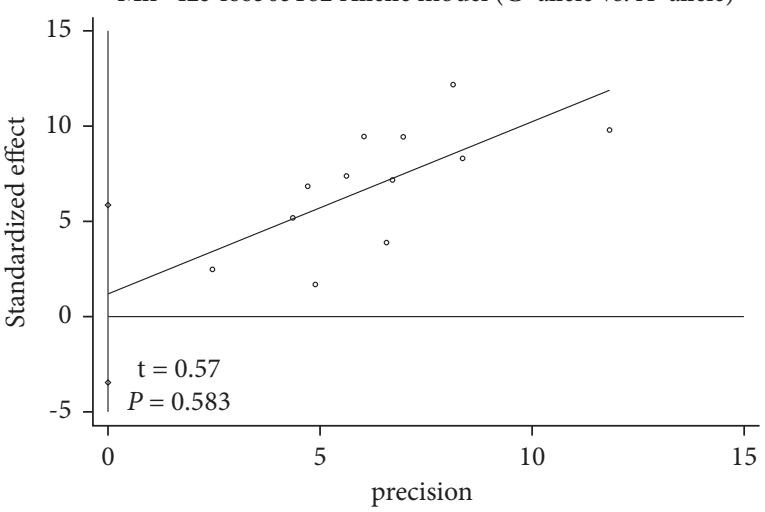

(b)

Egger's publication bias plot Mir-423 rs6505162 Recessive model (CC vs. AC + AA)

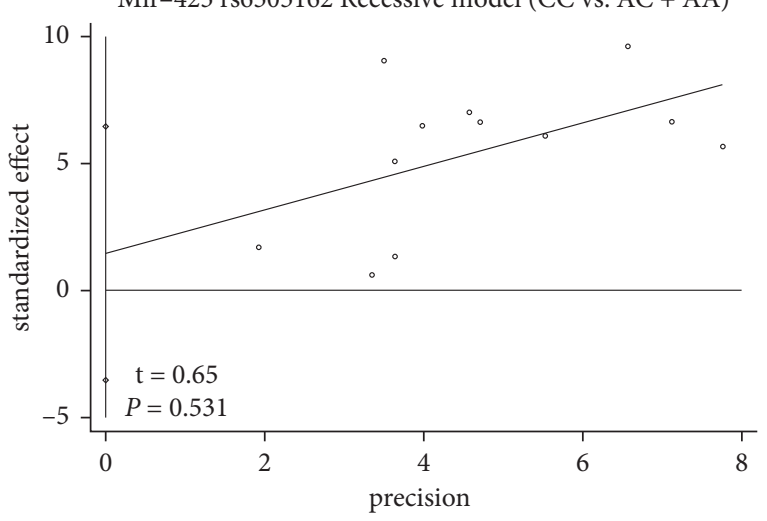

(d)

Egger's publication bias plot

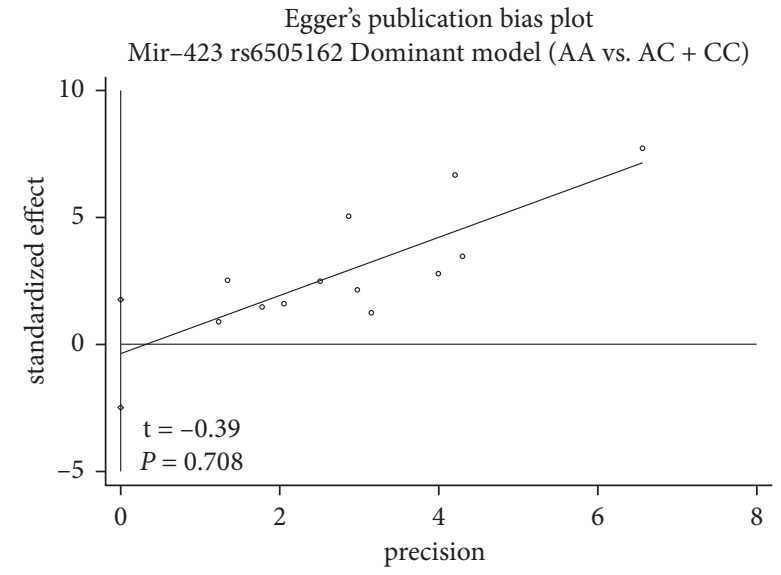

(f)

Figure 3: Continued. 


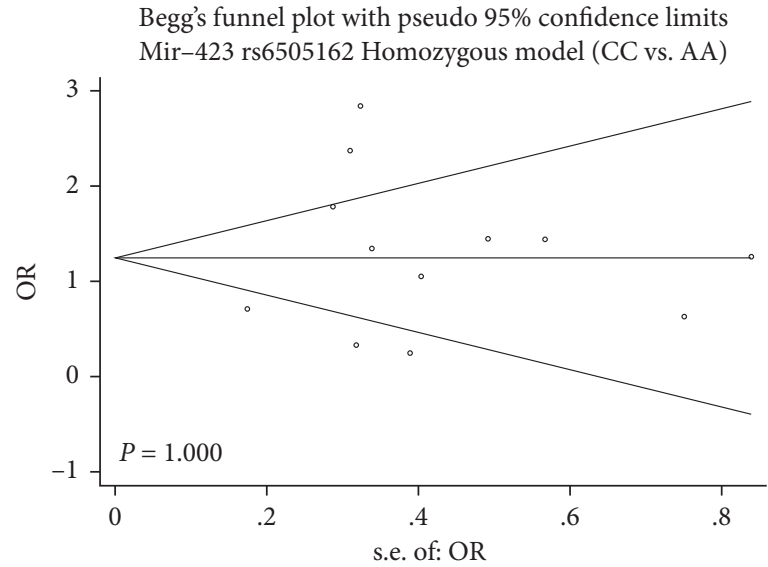

(g)

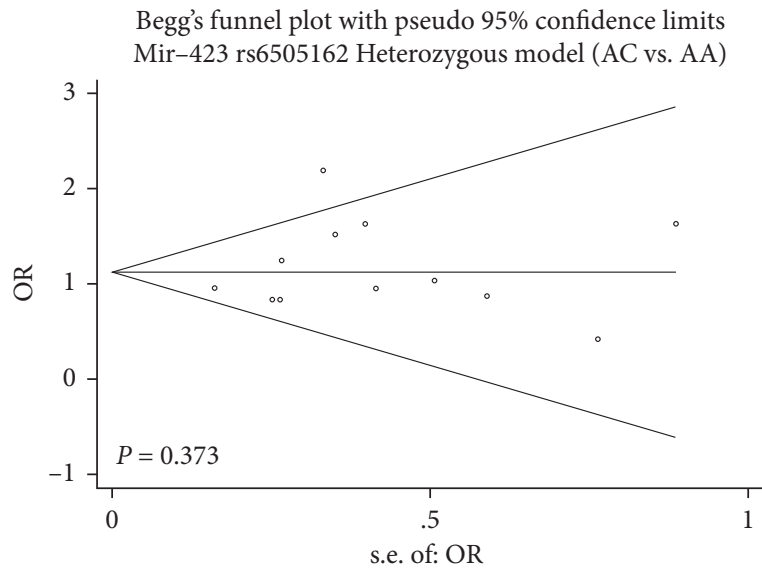

(i)

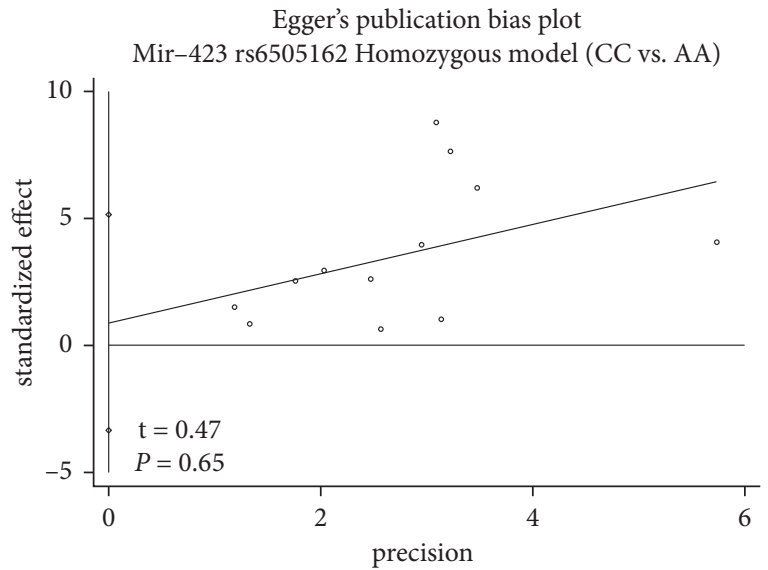

(h)

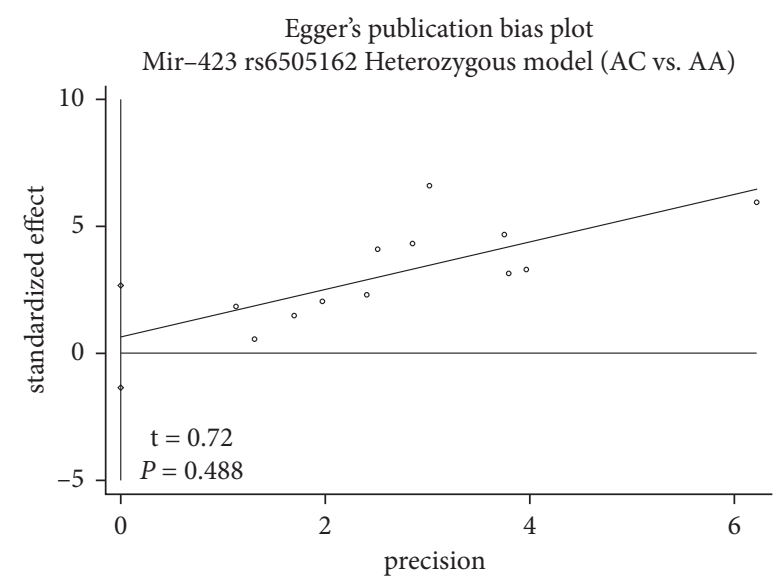

(j)

Figure 3: Begg's funnel plot (a) and Egger's test (b) to assess publication bias risk in analysis of the association between microRNA-423 rs6505162 polymorphism and breast cancer risk in total population according to all the genotype models.

In conclusion, this study performed an extensive assessment based on a larger sample size than the previous pooled analysis and suggested no significant association between miR-423 rs6505162 polymorphism and BC risk. These conclusions should be verified in large, well-designed studies.

\section{Abbreviations}

miR: $\quad$ MicroRNA

BC: $\quad$ Breast cancer

HWE: Hardy-Weinberg equilibrium

CNKI: Chinese National Knowledge Infrastructure

OR: $\quad$ Odds ratios

95\% CI: $95 \%$ confidence interval

TNBC: Triple-negative breast cancer

HB: $\quad$ Hospital-based source of control

PB: $\quad$ Population-based source of control

PCR: Polymerase chain reaction

RFLP: Restriction fragment length polymorphism

HRM: High-resolution melting

ARMS: Amplification refractory mutation system.

\section{Data Availability}

The data used to support the findings of this study are included within the article.

\section{Conflicts of Interest}

The authors declare that they have no conflicts of interest.

\section{References}

[1] M. F. Ullah, "Breast cancer: current perspectives on the disease status," Advances in Experimental Medicine and Biology, vol. 1152, pp. 51-64, 2019.

[2] K. McPherson, "Breast cancer-epidemiology, risk factors, and genetics," BMJ British Medical Journal, vol. 309, no. 7261, pp. 1003-1006, 1994.

[3] F. Qian, Y. Feng, Y. Zheng et al., "Genetic variants in microRNA and microRNA biogenesis pathway genes and breast cancer risk among women of African ancestry," Human Genetics, vol. 135, no. 10, pp. 1145-1159, 2016.

[4] S. Morales, T. De Mayo, F. Gulppi et al., "Genetic variants in pre-miR-146a, pre-miR-499, pre-miR-125a, pre-miR-605, and pri-miR-182 are associated with breast cancer 
susceptibility in a south American population," Genes, vol. 9, no. 9 , p. $427,2018$.

[5] M. R. Ladomery, D. G. Maddocks, and I. D. Wilson, "MicroRNAs: their discovery, biogenesis, function and potential use as biomarkers in non-invasive prenatal diagnostics," International Journal of Molecular Epidemiology and Genetics, vol. 2, no. 3, pp. 253-260, 2011.

[6] J. Lin, S. Huang, S. Wu et al., "MicroRNA-423 promotes cell growth and regulates $\mathrm{G} 1 / \mathrm{S}$ transition by targeting p21Cip1/ Waf1 in hepatocellular carcinoma," Carcinogenesis, vol. 32, no. 11, pp. 1641-1647, 2011.

[7] S. Morales, F. Gulppi, P. Gonzalez-Hormazabal et al., "Association of single nucleotide polymorphisms in Pre-miR-27a, Pre-miR-196a2, Pre-miR-423, miR-608 and Pre-miR-618 with breast cancer susceptibility in a south American population," BMC Genetics, vol. 17, p. 109, 2016.

[8] H. Zhao, A. Gao, Z. Zhang et al., "Genetic analysis and preliminary function study of miR-423 in breast cancer," Tumor Biology, vol. 36, no. 6, pp. 4763-4771, 2015.

[9] Q.-H. Chen, Q.-B. Wang, and B. Zhang, "Ethnicity modifies the association between functional microRNA polymorphisms and breast cancer risk: a HuGE meta-analysis," Tumor Biology, vol. 35, no. 1, pp. 529-543, 2014.

[10] G. A. Wells, B. Shea, D. O'Connell et al., "The newcastleottawa scale (NOS) for assessing the quality of nonrandomised studies in meta-analyses," 2000, http://www.ohri. $\mathrm{ca} /$ programs/clinical_epidemiology/oxford.htm.

[11] R. L. Ownby, E. Crocco, A. Acevedo, V. John, and D. Loewenstein, "Depression and risk for alzheimer disease," Archives of General Psychiatry, vol. 63, no. 5, pp. 530-538, 2006.

[12] Z. Hu, J. Liang, Z. Wang et al., "Common genetic variants in pre-microRNAs were associated with increased risk of breast cancer in Chinese women," Human Mutation, vol. 30, no. 1, pp. 79-84, 2009.

[13] K. M. T. Arif, G. Bradshaw, T. T. N. Nguyen et al., "Genetic association analysis implicates six MicroRNA-related SNPs with increased risk of breast cancer in Australian caucasian women," Clinical Breast Cancer, vol. S1526-8209, no. 21, 2021.

[14] F. Bahreini, E. Rayzan, and N. Rezaei, "microRNA-related single-nucleotide polymorphisms and breast cancer," Journal of Cellular Physiology, vol. 236, no. 3, pp. 1593-1605, 2021.

[15] G. Farnoosh, A. Saeedi-Boroujeni, A. Jalali, B. Keikhaei, and M.-R. Mahmoudian-Sani, "Polymorphisms in genes involved in breast cancer among Iranian patients," Personalized Medicine, vol. 18, no. 2, pp. 153-169, 2021.

[16] C. B. Zhu, Y. Q. Pan, and B. S. He, "Study on the association of polymorphisms in microRNAs and risk of breast cancer and its clinic pathological parameters," Medical Journal of Southeast China, vol. 17, no. 1, pp. 22-25, 2015.

[17] T. Kontorovich, A. Levy, M. Korostishevsky, U. Nir, and E. Friedman, "Single nucleotide polymorphisms in miRNA binding sites and miRNA genes as breast/ovarian cancer risk modifiers in Jewish high-risk women," International Journal of Cancer, vol. 127, no. 3, pp. 589-597, 2010.

[18] R. A. Smith, D. J. Jedlinski, P. N. Gabrovska, S. R Weinstein, L Haupt, and L. R Griffiths, "A genetic variant located in miR423 is associated with reduced breast cancer risk," Cancer Genomics and Proteomics, vol. 9, no. 3, pp. 115-118, 2012.

[19] F. Ma, P. Zhang, D. Lin et al., "There is no association between microRNA gene polymorphisms and risk of triple negative breast cancer in a Chinese Han population," PLoS One, vol. 8, no. 3, Article ID e60195, 2013.
[20] B. He, Y. Pan, Y. Xu et al., "Associations of polymorphisms in microRNAs with female breast cancer risk in Chinese population," Tumor Biology, vol. 36, no. 6, pp. 4575-4582, 2015.

[21] P. Zhang, F. Ma, and B. Xu, "Polymorphisms of microRNA genes and their association with breast cancer risk," Journal of Oncology, vol. 37, pp. 501-507, 2015.

[22] H. Zhao, A. Gao, Z. Zhang et al., "Genetic analysis and preliminary function study of miR-423 in breast cancer," Tumor Biology, vol. 36, no. 6, pp. 4763-4771, 2015.

[23] S. Saedi, S. Mashayekhi, Z. Salehi, S. Soltanipour, and E. Mirzajani, "The relationship between miR-423 genetic variation and women breast cancer risk in the north of Iran," Journal of Guilan University of Medical Sciences, vol. 26, no. 104, pp. 14-21, 2018.

[24] M. H. Tran Thi, T. Nguyen Thi Ngoc, and H. Nguyen Thi, "Association between selected microRNA SNPs and breast cancer risk in a Vietnamese population," International Journal of Human Genetics, vol. 18, pp. 238-246, 2018.

[25] R. Mir, I. A. Al Balawi, and F. M. A. Duhier, "Involvement of microRNA-423 gene variability in breast cancer progression in Saudi Arabia," Asian Pacific Journal of Cancer Prevention, vol. 19, no. 9, pp. 2581-2589, 2018.

[26] R. Mir, I. A. Al Balawi, and F. M. Abu-Duhier, "Role of microRNA-423 gene variation in women at higher risk of breast cancer in tabuk of Saudi Arabia," Indian Journal of Public Health Research \& Development, vol. 10, no. 2, 2019.

[27] N. Pourmoshir, G. H. Motalleb, and S. Vallian, "hsa-miR-423 rs6505162 is associated with the increased risk of breast cancer in isfahan central province of Iran," Cell Journal, vol. 22, no. S1, pp. 110-116, 2020.

[28] R. Chen, Y. Zheng, L. Zhuo, and S. Wang, "The association between miR-423 rs6505162 polymorphism and cancer susceptibility: a systematic review and meta-analysis," Oncotarget, vol. 8, no. 25, pp. 40204-40213, 2017.

[29] H. Zhang, Y. Zhang, X. Zhao et al., "Association of two microRNA polymorphisms miR-27 rs895819 and miR-423 rs6505162 with the risk of cancer," Oncotarget, vol. 8, no. 29, pp. 46969-46980, 2017.

[30] N. Li, Z. Cui, S. Li et al., "The association between two common polymorphisms and cancer susceptibility: a metaanalysis," Journal of Surgical Research, vol. 253, pp. 156-166, 2020. 